\title{
25 Research Suare \\ Ovarian non-gestational choriocarcinoma: a case report and review of current literature
}

\section{Ling Han}

Sichuan University WCSUH: Sichuan University West China Second University Hospital

\author{
Ai Zheng \\ Sichuan University West China Second University Hospital \\ Yali Chen ( $\boldsymbol{V}$ hanlingluobo@sina.com ) \\ Sichuan University West China Second University Hospital
}

\section{Case Report}

Keywords: ovarian choriocarcinoma, non-gestational, diagnosis, treatment, case report

Posted Date: June 24th, 2021

DOI: https://doi.org/10.21203/rs.3.rs-646074/v1

License: (c) (1) This work is licensed under a Creative Commons Attribution 4.0 International License. Read Full License 


\section{Abstract}

Background: Non-gestational ovarian choriocarcinoma is an extremely rare malignant tumor without established treatment. Because of the rarity of Non-gestational ovarian choriocarcinoma, limited information is available in the literature. Thus, we presented our case and reviewed other cases concerningtheir clinical characteristics, diagnosis, therapeutic effects, and prognoses using the PubMed database.

Case presentation: We present a rare case of a patient with Non-gestational ovarian choriocarcinoma with a dysgerminoma. Moreover, we performed a subsequent literature review.An 11-year-old Chinese girl presented with pain persisting for 20 days and a lump in her lower abdomen. Exploratory laparotomy was performed, and postoperative pathological sampling revealed a mixed germ-cell tumor comprising choriocarcinoma and dysgerminoma. Following six cycles of bleomycin, etoposide, and cisplatin, the patient remained recurrence-free after16 months from surgery.

Conclusions: The analysis of our and previously reported cases indicated that fertility-sparing surgery combined with postoperative chemotherapy might be beneficial for Non-gestational ovarian choriocarcinoma treatment in adolescent and young female individuals. Longer follow-up periods and further management data are necessary.

\section{Introduction}

Ovarian choriocarcinoma is a rare malignant tumor that may be gestational or non-gestational. Most ovarian choriocarcinomas arise from ovarian pregnancies or metastasize from uterine choriocarcinomas. Non-gestational ovarian choriocarcinomas (NGCOs) are exceedingly rare germ-cell tumors. Their prognosisis worse than that of gestational ovarian choriocarcinomas. Furthermore, it is important to distinguish between the two subtypes,as each has different chemosensitivity and prognosis. In the absence of confirmed pathological results, the diagnosis of NGCOs can be difficult in prepubertal female patients. The microscopic appearance of an NGCO is histologically characterized by the presence of mononucleated cytotrophoblastic and syncytiotrophoblastic cells. Herein, we present the case of a patient with mixed NGCO with dysgerminoma. Moreover, we have performed a brief literature review of such cases.

\section{Case Report}

An 11-year-old Chinese girl presented to our hospital with pain persisting for 20 days and a lump in her lower abdomen. There was no history of isosexual precocity,fever, nausea, vomiting, orloss of appetite or weight. The patient had experienced menarche six months prior to presentation, and her menstrual cycle was irregular.

Gynecological examination findings revealed a fixed, firm mass of approximately $20 \times 10 \mathrm{~cm}$ arising from the pelvis. Laboratory investigations revealed elevated serum levels of human chorionic 
gonadotropin(hCG) $(>200,000 \mathrm{mlU} / \mathrm{mL})$, alpha-fetoprotein $(39.3 \mathrm{ng} / \mathrm{mL})$, and cancer antigen (CA)-125 $(54.6 \mathrm{U} / \mathrm{mL})$. The carcinoembryonic antigen and CA19-9 levels were within the normal ranges. While the chest radiography findings were unremarkable,the ultrasound and computed tomography findings revealed the presence of a large, left-sided, lower abdominal pelvic mass extending to the level of the umbilicus, consistent with an ovarian tumor.

An exploratory laparotomy was performed at our hospital. Intraoperative assessment revealed that the right ovary had a $20 \times 10 \times 10 \mathrm{~cm}$ large solid tumor(Fig. 1). The left ovary and uterus were normal. There was no ascites. Further examination revealed no signs of extraovarian metastasis in the abdomen. Frozen-section examination during surgery revealed that the tumor was a malignant germ-cell tumor. Right salpingo-oophorectomy, total omentectomy, appendectomy, peritoneal washing, and peritoneal biopsies were performed.

Macroscopically, the right ovary mass was solid and firm,with gray/white cut surface and hemorrhage areas(Fig. 1A).Microscopically,the main component of the tumor was dysgerminoma accompanied by scattered mononucleated cytotrophoblastic and polynucleated syncytiotrophoblastic cells(Fig.

1B).Immunohistochemical stainingfindings showed dysgerminoma was positive staining for OCT3/4(Fig. 1C) and Sall-4(Fig. 1D). Both mononucleated cytotrophoblastic and syncytiotrophoblastic cells showed positive stains for AE1/AE3 (Fig. 1E), and syncytiotrophoblastic cells were highlighted by hCG(Fig. 1F).

After the surgery, the patient received six cycles of the BEP regimen consisting of bleomycin, etoposide, and cisplatin. The blood hCG levels dropped to normal two months postoperatively. The patient recovered well and had no recurrence 16 months after the surgery.Because of the short follow-up period, the longterm outcomes in this patient remain unclear.

\section{Discussion}

NGCO is an extremely rare malignant tumor that mostly occurs in women of reproductive age $[1,2]$. Nongestational choriocarcinomas commonly arise from germ cells and are not related to pregnancy. Most commonly,NGCOs are mixed and associated with other germ-cell neoplasms [3]. The present case involved choriocarcinoma coexisting with a dysgerminoma. Because of the rarity of NGCOs, limited information is available in the literature. Thus, we presented our case and reviewed other cases concerningtheir clinical characteristics, diagnosis, therapeutic effects, and prognoses using the PubMed database.We found 78 cases of ovarian NGCOs reported in the English literature[4-7]. Liu et al.reviewed 39 cases reported between January 1967 and July 2018[4]. In 2020, Shao et al.conducted a retrospective study, which reported 37 cases of NGCOs[5]. Moreover, another two similar cases were reported by Zhang et al.and Peng et al.in 2019 and2020, respectively[6-7].

NGCO usually occurs in patients who are sexually immature or virgins. The clinical presentation of NGCO is nonspecific and mostly includes vaginal bleeding, abdominal pain and distention, and pelvic masses.Liu et al. reported that the median age,primary tumor size, and serum hCG levels of 39patients 
with ovarian NGCOs were 30.4 years, $11.3 \mathrm{~cm}$, and 15,050IU/L, respectively. NGCO tends to metastasize early to the lungs, bowel, pelvic organs, and liver through blood-borne dissemination [4].

The preoperative diagnosis of NGCO is difficult. In the presence of an adnexal mass, abdominal pain, vaginal bleeding, and elevated hCG, NGCO can be easily mistaken for ectopic pregnancy in reproductiveaged women. In 1963, Saito et al. first reported the diagnostic criteria for NGCO, which included the absence of disease in the uterine cavity, pathological confirmation of disease, exclusion of molar pregnancy, and exclusion of a coexisting intrauterine pregnancy [8].In our case,

markedly elevated hCG levels and a pelvic mass are presented in a girl without a sexual experience and, the presence of a germ-cell tumor was considered first. According to Saito's criteria and considering that our patient was an 11-year-old girl with no previous sexual experience, the markedly elevated hCG levels and the pathological result comprised of mononucleated cytotrophoblastic cells and polynucleated syncytiotrophoblastic cells led us to the diagnosis of NGCO.It is difficult to differentiate gestational choriocarcinoma from an NGCO after performing the routine histological examination in patients ofreproductive age because the immunohistochemical features are the same. More recently, it was stated that DNA polymorphism analysis couldbe used to distinguish between the two tumor types $[9,10]$. DNA polymorphic analysis can verify the presence or absence of paternal DNA. We can make the final diagnosis of gestational choriocarcinoma when paternal DNA was present. DNA polymorphic analysis makes the diagnosis of NGCO easier in patients who had sexual intercourse.However, the cost of this method restricts its use.

In our case,the initial approach to the treatment of NGCO included laparotomy. The use of laparoscopy for ovarian tumors remains controversial. Xin et al. reported a positive oncologic outcome through laparoscopy; one of its advantages was the ability to allow rapid initiation of adjuvant chemotherapy [11]. However, the use of laparoscopy in NGCO appears to be limited, and there are few reports on the safety aspects of this approach.

To date, no standard treatment has been established for NGCO because of its low incidence. Liu et al. reported that $54 \%$ of cases received comprehensive staging surgery and chemotherapy; the remaining cases received fertility-preserving surgery and chemotherapy [4]. The 3-year overall survival rates for patients with FIGO stages I, II, III, and IV were $100 \%, 100 \%, 100 \%$, and $25 \%$,respectively [4]. Shao et al. reported 37 cases of NGCO that received surgery and a median of fourcourses of chemotherapy. The overall complete response rate, relapse rate, and 3- and 5-year survival rates were $81.1 \%, 16.7 \%$, and $80.0 \%$ and $75.5 \%$, respectively [5]. Goswami et al. and Kong et al. reported that the survival rates of patients who underwent surgery followed by chemotherapy were $81 \%$ and $86.1 \%$, respectively, and the survival rates of thosewho underwent surgery without chemotherapy were $28 \%$ and $33.3 \%$, respectively [3, 12]. Because NGCO occurs mostly in sexually immature and virgin patients, fertility-sparing surgery should be considered. There is no consensus on the optimal post-surgery chemotherapy because ofthe limited data on NGCOs. Choriocarcinoma responds well to a methotrexate-based regimen. Therefore, more aggressive combination chemotherapy (such as etoposide, methotrexate, and actinomycin-D 
administration)has been used in several reported cases. Non-gestational choriocarcinoma is considered to arise from a germ-cell tumor. Therefore, NGCOs may be resistant to these chemotherapy protocols. Furthermore, BEP and PVB(cisplatin, vinblastine, andbleomycin) regimens have also been administered. The current patient underwent conservative surgery and postoperative chemotherapy,resulting in a favorable decline in postoperative hCG levels. An analysis of our case and those reported in previous case studies suggested that NGCO responds well to surgery combined with chemotherapy.

\section{Conclusions}

Because of its rarity, there is no consensus on the management of NGCO. We reported fertility-sparing surgery combined with cisplatin-based chemotherapy was used in this rare disease,which appears to be beneficial for the treatment of NGCO in adolescent and young female patients. The case which we reported adds data that can be helpful in both diagnosis and treatment of ovarian NGCO in the future. Accumulation of more data on the management of these rare tumors and a long-term follow-up examination period in such patients is important.

\section{Abbreviations}

NGCO, non-gestational ovarian choriocarcinoma; hCG, human chorionic gonadotropin; CA, cancer antigen; CT, computed tomography; EMA, etoposide, methotrexate, actinomycin-D; BEP, bleomycin, etoposide, and cisplatin; PVC,cisplatin, bleomycin, and vinblastine.

\section{Declarations}

\section{Ethics approval and consent to participate}

The study was approved by the ethics committee of the West China Second University Hospital. The patient provided written informed consent for the publication of his case details.

\section{Authors' contributions}

Han Ling was responsible for the conception of the paper and manuscript drafting. Zheng Ai and Chen YaLi contributed to the revision and final approval of the manuscript.

\section{Conflicts of Interest}

The authors declare no conflicts of interest.

\section{Acknowledgements}

We thank all the peer reviewers for their opinions and suggestions. We thank Dr. Sun Liang who provided the figures for our manuscript. 


\section{Funding}

The study was funded by the Sichuan Science and Technology Program (No.2017SZ0118 and No.19ZDYF1743.)

\section{Consent for publication}

Written informed consent for publication was obtained from all participants.

\section{Availability of data and materials}

Not applicable

\section{References}

1. Corakçi A, Ozeren S, Ozkan S, GürbüzY, UstünH, Yücesoyl. Pure nongestational choriocarcinoma of ovary. Archives of Gynecology and Obstetrics. 2005; 271: 176-177.

2. Exman P, Takahashi TK, Gattás GF, Cantagalli VD, Anton C, Nalesso F, et al. Primary ovary choriocarcinoma: individual DNA polymorphic analysis as a strategy to confirm diagnosis and treatment. Rare Tumors. 2013; 5: 89-92.

3. Goswami D, Sharma K, Zutshi V, Tempe A, Nigam S. Nongestational pure ovarian choriocarcinoma with contralateral teratoma. Gynecological Oncology. 2001; 80:262-266.

4. Liu X, Zhang X, Pang Y, Ma Y, Zhang X, Liu P. Clinicopathological factors and prognosis analysis of 39 cases of non gestational ovarian choriocarcinoma. Archives of Gynecology and Obstetrics. 2020; 301: 901-912.

5. Shao Y, Xiang Y, Jiang F, Pan B, Wan X, Yang J, et al. Clinical features of a Chinese female nongestational choriocarcinoma cohort: a retrospective study of 37 patients. Orphanet Journal of Rare Diseases. 2020; 15: 1-7.

6. Zhang X, Yan K, Chen J, Xie X.Using short tandem repeat analysis for choriocarcinoma diagnosis: acase series. Diagn Pathol.2019;14:93.

7. Peng $\mathrm{H}$, Li L, Bi Y. Successful management of nongestational ovarian choriocarcinoma complicated with choriocarcinoma syndrome: A case report and a literature review.CurrProbl Cancer. 2020;44:100539.

8. Saito M, Azuma T, Nakamura K. On ectopic choriocarcinoma. World Journal of Obstetrics and Gynecology. 1965; 17: 459-484.

9. Tsujioka H, Hamada H, Miyakawa T, Hachisuga T, Kawarabayashi T. A pure nongestationalchoriocarcinoma of the ovary diagnosed with DNA polymorphism analysis. Gynecological Oncology. 2003; 89: 540-542.

10. Shigematsu T, Kamura T, Arima T, Wake N, Nakano H. DNA polymorphism analysis of a pure nongestational choriocarcinoma of the ovary: case report. European Journal of Gynecological Oncology. 
2000; 21: 153-154.

11. Xin L, Beier A, Tiede S, Pfiffer T, Köhler C, Favero G. Laparoscopic fertility-preserving treatment of a pure nongestationalchoriocarcinoma of the ovary: case report and review of current literature. Journal of Minimally Invasive Gynecology. 2015; 22: 1095-1099.

12. Kong B, Tian YJ, Zhu WW, Qin YJ. A pure nongestationalovarian choriocarcinoma in a 10-year-old girl: case report and literature review. Journal of Obstetrics and Gynaecology Research. 2009; 35 : 574-578.

\section{Figures}

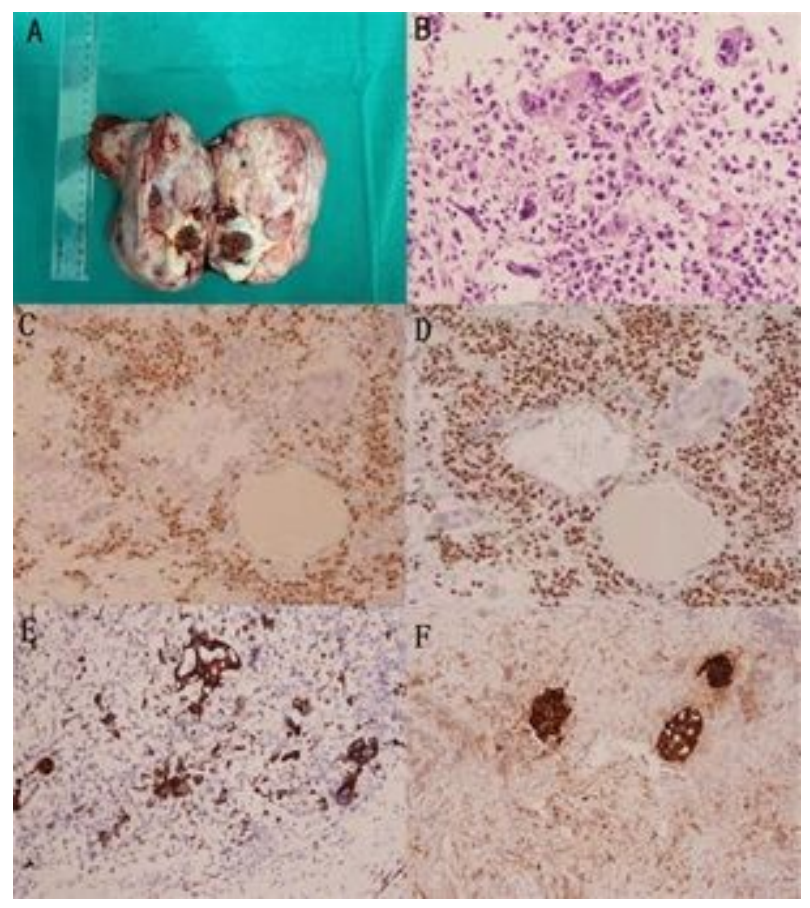

\section{Figure 1}

A. The right ovary mass showed gray/white cut surface with hemorrhage area.B.The histological components of the tumor showed a dysgerminoma with scattered mononucleated cytotrophoblastic cells and syncytiotrophoblastic cells (×200).C-D.Dysgerminoma showedpositive immunohistochemical staining for OCT3/ 4(Fig.C) and Sall-4(Fig.1D) (×100). E.Both mononucleated cytotrophoblastic and syncytiotrophoblastic cells showed positive immunohistochemical staining for AE1/AE3(×100).F. Syncytiotrophoblastic cells showed positive immunohistochemical staining for human chorionic gonadotropin $(\times 100)$. 